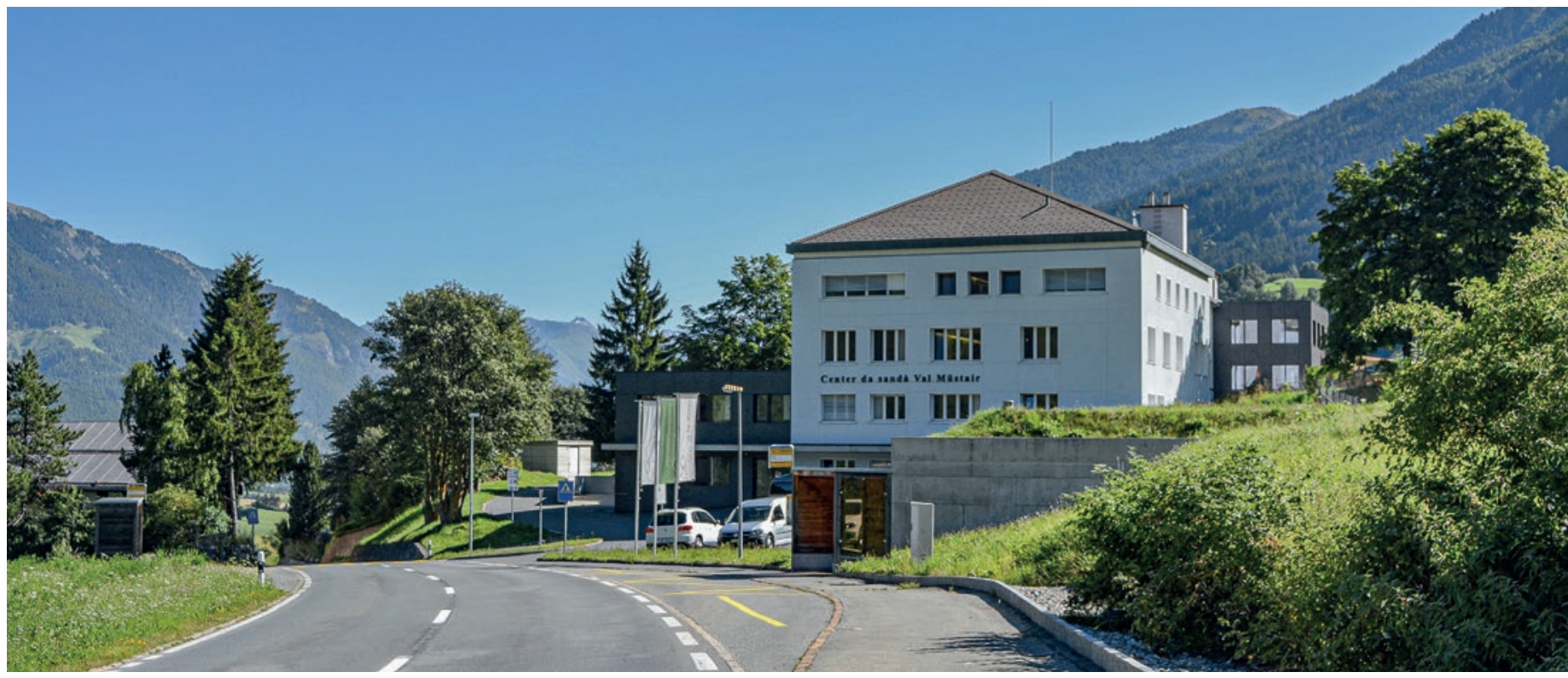

Das Center da sandà im Val Müstair, Kanton Graubünden.

\title{
Medizinische Versorgung in peripheren Regionen
}

\section{Sébastien Jotterand}

Präsident der Plattform Interprofessionalität in der medizinischen Grundversorgung, Vizepräsident Haus- und Kinderärzte Schweiz (mfe)

Im Kampf gegen den Pflegenotstand kommt den Gemeinden eine wichtige Rolle zu, vor allem in den Rand- und Bergregionen. Zu dieser Feststellung kam ein jüngst von der Plattform Interprofessionalität organisiertes Symposium mit dem Titel «Interprofessionelle Zusammenarbeit als Garant für den Zugang zur medizinischen Grundversorgung in peripheren Regionen?».

Wenn wir von Interprofessionalität sprechen, denken wir zunächst vor allem an den Meinungsaustausch innerhalb eines Pflegeteams über und mit der jeweiligen Patientin resp. dem jeweiligen Patienten, um so das Gesundheitsproblem besser und umfassender zu verstehen. Wir wissen, dass eine möglichst genaue Darstellung der gesundheitlichen Problematik einer Patientin bzw. eines Patienten wesentlich zur Lösungsfindung beiträgt.

Interprofessionalität bringt auch quantitative Vorteile. Im Bereich der Grundversorgung zeigt sich beispielsweise ein erheblicher Mangel an Gesundheitsfachpersonen, insbesondere ein Mangel an Hausärztinnen und Hausärzten. Gemäss einer von Prof. Andreas Zel- ler von der Universität Basel durchgeführten Studie wird sich erst nach 2030 - nach dem Wegfall der BabyBoomer - messen lassen, wie sich die grössere Zahl von Medizinstudierenden auf die Nachfolge auswirkt [1]. Daher gilt es, ab sofort gemeinsam darüber nachzudenken, wie eine neue Rollenverteilung zwischen den

Schwerpunktserie Interprofessionalität

Die interprofessionelle Zusammenarbeit von Fachpersonen aus verschiedenen Gesundheitsberufen gilt als wichtiges Mittel, um den Herausforderungen im Gesundheitswesen zu begegnen. Aber wie weit ist die Schweiz in diesem Bereich tatsächlich? Welche Hürden und Chancen gibt es? In unserer Schwerpunktserie betrachten wir dasThema aus unterschiedlichen Perspektiven. 
in der Grundversorgung tätigen Akteurinnen und Akteuren diesen Mangel beheben könnte.

Zwar sieht unsere Verfassung vor, dass die Kantone und der Bund den Zugang zur Grundversorgung zu gewährleisten haben, doch im Kampf gegen den Pflegenotstand kommt aus unserer Sicht auch den Gemeinden eine wichtige Rolle zu, vor allem in den peripheren Regionen und Bergregionen.

\section{Rettung des Regionalspitals}

Das beste Beispiel dafür in der Schweiz ist das Münstertal in Graubünden. Der Zugang zum Tal ist sehr schwierig. Bei schlechtem Wetter ist er gesperrt. Im Tal wohnen 1500 Menschen, was kaum die Anwesenheit einer Ärztin oder eines Arztes rechtfertigt. Und dennoch ist es dank einer regionalen Mobilisierung der

\section{Wie könnte eine neue Rollenverteilung zwi- schen den in der Grundversorgung tätigen Akteuren den Fachkräftemangel beheben?}

zuständigen Behörden und örtlichen Akteure aus dem Gesundheitsbereich gelungen, nicht nur das regionale Spital und die damit verknüpften medizinischen und paramedizinischen Dienste zu retten, es konnte sogar noch ein Gesundheitszentrum mit spezieller Pflege für alte Menschen vor Ort und zu Hause ins Leben gerufen werden. Die dort bereits seit mehr als einem Vierteljahrhundert beschäftigten Mitarbeitenden zeigten sich begeistert und zufrieden, dass es ihnen gelungen ist, die Kontinuität der Pflege für die Bewohnerinnen und Bewohner ihrer Region zu gewährleisten.

\section{Wie geht das?}

Angesichts der saisonal variierenden Nachfrage wurden der Spitaldienst und die ambulanten Dienste des Gesundheitszentrums entsprechend reguliert. Im Krisenfall verteilen sich die Einsatzkräfte nach dem Prinzip der kommunizierenden Röhren. Die Überbelastungsphasen sind dieselben, die wir auch im Praxisalltag erleben und die uns eine andere Aufgabenverteilung abverlangen. Dies geht von der Bewertung der Kompetenzen in der medizinischen Assistenz und in der Koordination der ambulanten Medizin über den Einbezug von Pflegeteams für die Pflege zu
Hause zur Erstevaluierung oder die feinjustierte telefonische Therapie unter Einbezug der jeweiligen Apotheke vor Ort.

\section{Umstrukturierung der Aufgabenstellung}

Diese neue Aufgabenverteilung erlebten wir doch alle bereits in der Covid-19-Krise, unter anderem auch in unseren zentralisierten und urbanen Regionen. So packten beispielsweise in meiner Region um Aubonne betroffene Geriaterinnen und Geriater von geschlossenen Memory Clinics mit an und stellten sich in den Dienst von Covid-19-Teams in unserem Akutspital in Morges. Kolleginnen und Kollegen von Gesundheitszentren in der Stadt halfen in Reha-Einrichtungen aus, als ein Corona-Cluster die Hälfte eines Pflegeteams in die häusliche Quarantäne verbannt hatte. Die Arbeitskräfte wandern dahin, wo sich der Bedarf zeigt. Gleiche Muster zeichnen sich ab, wenn Patientinnen oder Patienten aufgrund einer Polymorbidität oder bedingt durch soziale, psychische oder medizinische Vulnerabilitäten multiple Bedürfnisse zeigen. Für uns alle - ob Akteure im Gesundheitswesen oder pflegende Angehörige - gilt es daher, einen gemeinsamen Weg zu finden, um die Patientinnen und Patienten dabei zu unterstützen, Kompetenzen, die sie für ein Leben mit der Krankheit benötigen, zu erhalten oder zurückzugewinnen.

\section{Bildnachweis}

CSVM

\section{Literatur}

1 Zeller A, Giezendanner S. Resultate der 4. Workforce Studie. Prim Hosp Care Allg Inn Med. 2020;20(11):325-8. primary-hospital-care. ch/article/doi/phc-d.2020.10311

\section{Plattform Interprofessionalität}

Die Plattform Interprofessionalität wurde im Jahr 2018 gegründet. Sie ist das Ergebnis eines langen Arbeitsweges, der im Jahr 2013 als Initiative des Verbandes der Haus- und Kinderärzte Schweiz (mfe) seinen Anfang nahm und in dessen Verlauf es gelang, alle wichtigen Berufsverbände der medizinischen Grundversorgung in der Schweiz zu vereinen. Die erste Aufgabe der Plattform lag in der Sicherung der Qualität der interprofessionellen Zusammenarbeit, insbesondere durch die Auflistung entsprechender Qualitätskriterien. Gleichzeitig gilt es auch, die Arbeit der letzten Jahre gezielt fortzusetzen und sich vermehrt in die politische Diskussion einzubringen. 


\section{Center da sandà Val Müstair}

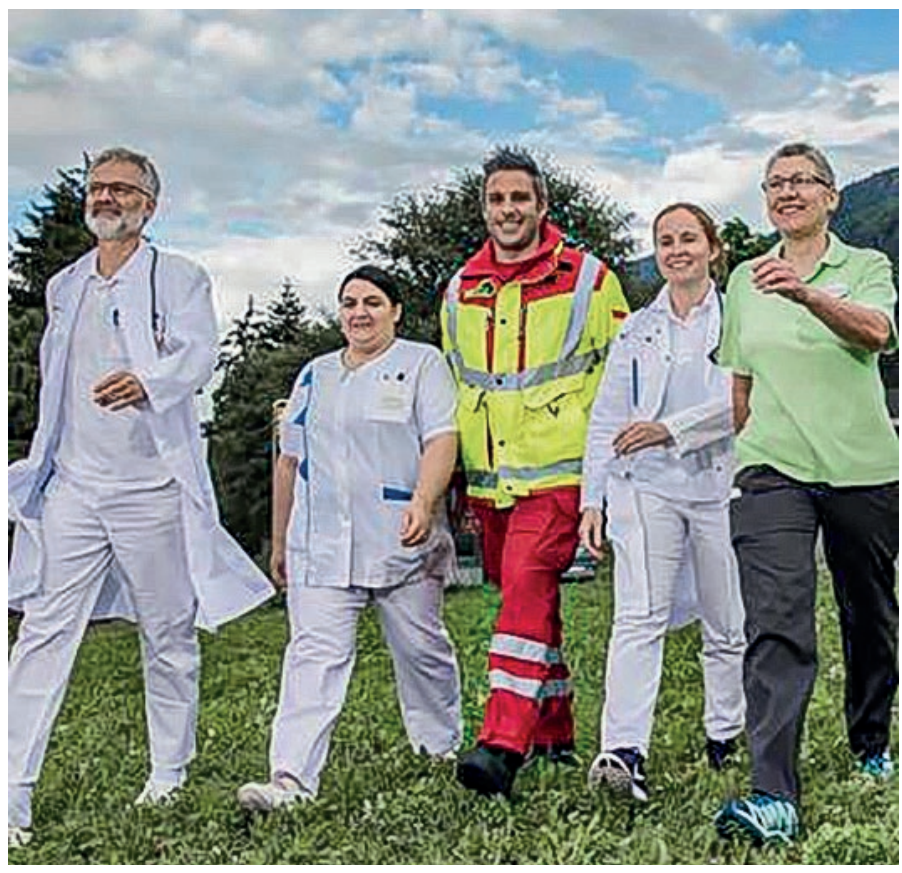

Um die Gesundheitsversorgung in einer Bergregion zu erhalten und möglichst effektiv und umfassend zu gestalten, müssen die verschiedenen Akteure eng zusammenrücken. Das ist im Val Müstair gelungen. Die Bereiche Arztpraxis, Spital, Pflegeheim, Spitex und Rettungsdienst sind unter einem Dach, dazu noch weitere Dienstleister. Die interprofessionelle Zusammenarbeit hat sich damit vereinfacht und vertieft. Alle Disziplinen bemühen sich gemeinsam um eine bestmögliche Notfall- und Grundversorgung, aber auch um ein gutes Leben zu Hause oder im Heim für die Patientinnen und Patienten. Unser Ziel ist eine optimale zeitnahe Betreuung im ambulanten und stationären Bereich vom Kleinkind bis zu den letzten Lebenstagen.

\section{Was wird damit erreicht?}

Die Patientinnen und Patienten stehen mit ihrer Gesundheit, aber auch ihren Bedürfnissen im Mittelpunkt. Mit dem Center da sandà haben sie einen primären Ansprechpartner für alle medizinischen Belange. Ein besonderer Vorteil sind die ärztliche Kontinuität als Hausärztinnen und Hausärzte, Spitalärztinnen und Spitalärzte sowie Heimärztinnen und Heimärzte sowie die Dokumentation in einem System. Ein weiterer Pluspunkt sind die kurzen Informationswege. Zentral für eine Behandlung ohne Unterbrüche ist unser einheitliches Qualitätsmanagement über alle Bereiche. Belegungsund Auslastungsschwankungen können wir rasch und unkompliziert begegnen. Einige Mitarbeitende, allen voran die Ärztinnen und Ärzte, aber auch der Rettungsdienst und einige Pflegende arbeiten in zwei oder mehreren Bereichen mit. Sie sind Brückenbauende für die Kontinuität und den Infofluss.

\section{Erste Resultate}

Das Austrittsmanagement vom Spital wird in der interdisziplinären Visite besprochen. Aufgaben des Case Managements werden dem bestgeeigneten Versorgungsbereich zur Klärung und Initiierung der Nachsorge zugeteilt. Ein gutes Beispiel dafür, dass die Beziehung und die Kontinuität eine wichtige Rolle spielen.

Darüber hinaus werden Palliativsituationen zu Hause ermöglicht. Neben der Hausärztin resp. dem Hausarzt und der Pflege sowie den freiwilligen Personen, die in Krisensituationen begleiten, werden auch Fachpersonen mit Zusatzausbildung in Palliative Care beigezogen. Pflegebett und Mahlzeiten können geliefert, der RotkreuzFahrdienst kann organisiert und die Wäsche kann im Center da sandà besorgt werden. Damit soll eine qualitativ hochstehende Rundumversorgung der Betroffenen gewährleistet werden.

\section{Judith Fasser, Direktorin/CEO Center da sandà, Val Müstair judith.fasser[at]csvm.ch}

Bildnachweis

CSVM

\section{Projekt PRiMA der Berner Fachhochschule}

Die Zunahme chronischer Erkrankungen, Multimorbidität sowie Fachkräftemangel machen eine Anpassung der Primärversorgung unabdingbar. Neue Versorgungsmodelle sind nötig, die sich an den Patientenbedürfnissen orientieren und somit umfassende Betreuung von chronisch Kranken sicherstellen. Die Ziele des Projekts PRiMA unter der Leitung von Prof. Dr. Maya Zumstein-Shaha sind es, das Potenzial von Advanced Practice Nurses (APN) bei der Versorgung von chronisch Kranken zu untersuchen, die interprofessionelle Zusammenarbeit darzustellen und Schnittstellen zu identifizieren. Zudem sollen Hinweise auf finanzielle Gegebenheiten abgeleitet werden, so dass die interprofessionelle Zusammenarbeit besser gestaltet werden kann. Das Projekt läuft von Januar 2020 bis März 2022.

\section{Projektaufbau}

Mittels eines Case-Study-Designs wurden zwei APN-Rollen, angesiedelt in zwei Hausarztpraxen im Kanton Bern (Mittelland und Bergregion), untersucht. Dabei kamen qualitative und quantitative Methoden zur Anwendung. Mittels Within-Case-Analyse wurden die Daten induktiv zu Fallgeschichten zusammengeführt. 


\section{Erste Ergebnisse}

Erste Ergebnisse zeigen, dass APN einen Mehrwert in den Bereichen Unterstützung des Selbstmanagements, Prävention und Gesundheitsförderung bei komplexen, stabilen und instabilen Patientensituationen leisten, die bis jetzt durch andere Gesundheitsberufe in den Hausarztpraxen nur unzureichend abgedeckt wurden.

Gerade bei Hausbesuchen stärken APN die Verbindung zwischen Patientinnen und Patienten, Angehörigen, Hausarztpraxis, Spitex oder anderen Leistungserbringern. Dabei ermöglichen sie transparente Kommunikation und bessere Koordination. Mit zunehmender Erfahrung der APN steigt ihr Selbstvertrauen sowie das Vertrauen der Ärzteschaft. Entsprechend ist das Niveau an Selbständigkeit im Wandel und wächst im Laufe einer Arbeitsbeziehung.
Diese Beziehung beeinflusst auch die Vergütung: APN sind im heutigen System nicht als Leistungserbringende vorgesehen. Daher fehlen eigene Tarife. Es bestehen Möglichkeiten, mittels bestehender Tarife im TARMED (nicht-ärztliche Fachpersonen) gewisse Leistungen innerhalb der Hausarztpraxis teilweise abzurechnen. Um den Einsatz von APN in Hausarztpraxen zu stärken, müssen deren Kompetenzen geklärt und eine generische Stellenbeschreibung erarbeitet werden.

Margarithe Charlotte Schlunegger, Wissenschaftliche Mitarbeiterin am Departement Gesundheit der Berner Fachhochschule, PhD Candidate an der Universität Witten/Herdecke margarithe.schlunegger[at]bfh.ch

\section{Projekt INSPIRE der Universität Basel}

Das Forschungsprojekt INSPIRE unter der Leitung von Prof. Dr. Sabina M. De Geest wurde dazu eingeladen, mit dem Kanton BaselLandschaft bei der Entwicklung eines Versorgungsmodells zusammenzuarbeiten, das auf vorhandener Evidenz basiert und mit dem aktuellen gesetzlichen Rahmen übereinstimmt. Im Jahr 2018 hat der Kanton Basel-Landschaft mit dem "Altersbetreuungs- und Pflegegesetz" (SGS 941) einen gesetzlichen Rahmen zur Förderung des "Aging in Place» geschaffen. Dieser schreibt die Neuorganisation des Kantons in Versorgungsregionen vor sowie die Einrichtung einer Informations- und Beratungsstelle (IBS) in den neu gebildeten Versorgungsregionen [1].

\section{Entwicklung eines Versorgungsmodells}

In diesem Zusammenhang entwickelte INSPIRE ein integriertes Versorgungsmodell für zu Hause lebende ältere Personen, das in zwei Versorgungsregionen des Kantons umgesetzt und evaluiert werden soll: in der Region ABS (Allschwil, Binningen, Schönenbuch) und im Leimental (Biel-Benken, Bottmingen, Burg im Leimental, Ettingen, Oberwil und Therwil). Ein Novum von INSPIRE ist die Anwendung eines implementierungswissenschaftlichen Ansatzes, um die Umsetzung in der Praxis zu unterstützen.

Dieser Ansatz baut auf den Methoden der klinischen Forschung auf, fügt jedoch die folgenden methodischen Elemente hinzu: wissenschaftliche Evidenz [2], Einbeziehung von Stakeholdern, Kontextanalyse [3], Implementierungsstrategien, doppelter Fokus auf Implementierungs- und Effektivitätsergebnisse und die Verwendung von Hybriddesigns. Zu den Stakeholdern gehören das Gesundheitsdepartement des Kantons Basel-Landschaft, Vertreterinnen und Vertreter von Leistungserbringern (z.B. Spitex), von Hausärztinnen und Hausärzten, von Pflegefachpersonen und Sozialarbeiterinnen und Sozialarbeitern. Als Ergebnis wurde ein Versorgungsmodell mit vier Komponenten erstellt:

1. Screening auf Gebrechlichkeit

2. Durchführung eines umfassenden geriatrischen Assessments zur Ermittlung von Bedürfnissen, Präferenzen und Pflegezielen
3. Entwicklung eines individuellen Versorgungsplans, der mit allen an der Pflege und Betreuung der Person beteiligten Leistungserbringern koordiniert wird

4. Erarbeitung einer bedarfsgerechten Nachsorge

\section{Begleitete Umsetzung in der Praxis}

Das Versorgungsmodell wird von einer Pflegefachperson in Zusammenarbeit mit einer Sozialarbeiterin oder einem Sozialarbeiter in den IBS der teilnehmenden Versorgungsregionen umgesetzt. Zur Evaluierung wird eine Machbarkeitsstudie durchgeführt. Daran schliesst sich eine hybride Implementierungs-Effektivitäts-Studie des Typs 1 an, um das Versorgungsmodell im Hinblick auf das Niveau der personenzentrierten und koordinierten Pflege sowie auf die Wirksamkeitsergebnisse (z.B. funktionaler Status, gesundheitsbezogene Lebensqualität und Pflegeheimeinweisungen) zu bewerten. Zusätzlich werden mit der Evidenz und dem Input der Stakeholder Prozesse konzipiert und evaluiert, um Förderfaktoren und Barrieren des implementierten Versorgungsmodells zu identifizieren. Die Zusammenarbeit mit den Stakeholdern soll die Akzeptanz und den Erfolg des Projekts sicherstellen.

Dr. med. Maria José Mendieta, PhD Candidate am Institut für Pflegewissenschaft der Universität Basel für das INSPIRE-Konsortium mariajose.mendietajara[at]unibas.ch

Literatur

1 Kanton Basel-Landschaft. 941 Altersbetreuungs- und Pflegegesetz (APG). 2018 http://bl.clex.ch/app/de/texts of law/941/versions/2126

2 Deschodt M, Laurent G, Cornelissen L, Yip O, Zúñiga F, Denhaerynck K, et al. Core components and impact of nurse-led integrated care models for home-dwelling older people: A systematic review and meta-analysis. Int I Nurs Stud. 2020;105:103552.

3 Yip O, Huber E, Stenz S, Zullig LL, Zeller A, De Geest SM, et al. A Contextual Analysis and Logic Model for Integrated Care for Frail Older Adults Living at Home: The INSPIRE Project. Int J Integr Care. 2021;21:9. 